\title{
Higgs induced light leptoquark-diquark mixing and proton decay
}

\author{
Uma Mahanta \\ Mehta Research Institute \\ Chhatnag Road, Jhusi \\ Allahabad-211019, India \\ email:mahanta@mri.ernet.in
}

\begin{abstract}
In low energy phenomenology to avoid the strong constraints of proton decay it is usually assumed that light $(\approx 250 \mathrm{Gev})$ leptoquarks couple only to quark-lepton pairs and light diquarks couple only to quark pairs. In this paper we present two specific examples where the higgs induced mixing between leptoquarks and diquarks through trilinear interaction terms reintroduces the troublesome couplings and gives rise to proton decay. The bound on the unknown parameters of this scenario that arise from proton life time has also been derived.
\end{abstract}


Leptoquarks (LQ) [1] and diquarks (DQ) [2, 3] are colored scalar or vector particles that carry baryon numbers of $\pm \frac{1}{3}$ and $\pm \frac{2}{3}$ respectively. They occur naturally in many extensions of the standard model (SM) e.g. superstring inspired grand unified models based on $E(6)$ [3], technicolor models [2] and composite models [1] of quarks and leptons. If LQs and DQs couple both to quark-lepton pairs and quark pairs then they lead to too rapid proton decay. To be consistent with the proton life time such LQs and DQs must have a mass of the order of $\left(10^{12}-10^{15}\right)$ Gev. To avoid this strong constraint and to make them relevant for low energy phenomenology below $1 \mathrm{Tev}$ it is usually assumed that LQs couple to quark-lepton pairs but not to quark pairs and DQs couple to quark pairs but not to quark-lepton pairs. However in the presence of the SM higgs doublet $\phi$ the low energy effective Lagrangian will also contain a trilinear Higgs-LQ-DQ interaction term. After EW symmetry breaking (EWSB) this interaction term will induce a mixing between the LQ and the DQ which reintroduces the troublesome Yukawa couplings for LQ and DQ. If the physical LQ and DQ do not have the same mass then they lead to proton decay. There can be several different kinds [1-3] of LQ's and DQ's. However in this report we shall not go into a detailed discussion of every LQ-DQ mixing that can possibly occur. Rather we shall give two give specific examples of Higgs-LQ-DQ interaction term and the mixing between the LQ and DQ that takes place after EWSB. For one particular case we have also calculated the mixing angle in terms of the dimensional coupling that measures the strength of the Higgs-LQ-DQ interaction term. Finally we have estimated the proton decay rate in terms of the unknown parameters of our scenario and have derived the constraint that the parameters of the Lagrangian must satisfy in order to be consistent with proton lifetime.

Consider the SM to be extended by a light $(\approx 250 \mathrm{Gev})$ chiral leptoquark D and a light chiral diquark $\mathrm{S}$ with the following assignments under $S U(3)_{c} \times S U(2)_{l} \times U(1)_{y}$ : $D \sim\left(3^{*}, 2,-\frac{1}{6}\right)$ and $S \sim\left(3^{*}, 1, \frac{1}{3}\right)$. The low energy effective Lagrangian of this extended scenario will contain besides the SM Lagranigian all possible renormalizable and gauge 
invariant interaction terms between $\mathrm{D}, \mathrm{S}$ and the SM fields. Of particular importance for this work are the Yukawa like couplings of D and S to the SM fermions given by the following Lagrangian

$$
\begin{aligned}
L_{y} & =g_{R} \bar{l}_{L} d_{R} D+g^{\prime} \epsilon_{i j k} \bar{d}_{R i} u_{R j}^{c} S_{k}+\text { h.c. } \\
& =g_{R}\left(\bar{\nu}_{L} D_{1}+\bar{e}_{L} D_{2}\right) d_{R}+g_{R}^{\prime} \epsilon_{i j k} \bar{d}_{R i} u_{R j}^{c} S_{k}+\text { h.c. }
\end{aligned}
$$

Here $D_{1}$ and $D_{2}$ are the isospin up and down components of D. i, j, k are the color indices. Note first that the leptoquark D couples only to quark-lepton pair and the diquark $\mathrm{S}$ couples only to quark pair which is required so that they do not lead to proton decay. Second both interaction terms are invariant under the SM gauge group $S U(3)_{c} \times S U(2)_{l} \times$ $U(1)_{y}$. Finally the couplings of $\mathrm{D}$ and $\mathrm{S}$ are both chiral in nature i.e. they couple to quark fields of a particular chirality only. The Yukawa Lagrangian $L_{y}$ also implies that D and $\mathrm{S}$ should carry baryon numbers of $-\frac{1}{3}$ and $\frac{2}{3}$ respectively so that $L_{y}$ conserves baryon number. This kind of structure of the Yukawa couplings could arise depending on the gauge symmetry of the high energy theory and its chiral matter representation. Besides the Yukawa couplings the low energy Lagrangian for describing physics much below the compositeness scale or grand unified scale $\Lambda$ must also contain all possible renormalizable and gauge invariant interaction terms between $\phi, \mathrm{D}$ and $\mathrm{S}$. In principle such interaction terms cannot be neglected. They could arise from the scalar potential of the high energy theory after the gauge symmetry of the high energy theory breaks down into $S U(3)_{c} \times S U(2)_{l} \times U(1)_{y}$. The gauge invariant trilinear interaction term between $\phi$, $\mathrm{D}$ and $\mathrm{S}$ is given by the Lagrangian

$$
L_{s}=k_{1}\left(D^{+} \phi_{c}\right) S+\text { h.c. }=k_{1} \frac{v+h}{\sqrt{2}} D_{1}^{+} S+\text { h.c. }
$$

Here $\phi_{c}$ is the charge conjugated Higgs doublet that gives mass to the up quarks in SM. The unknown mixing parameter $k_{1}$ carries the dimension of mass. After EW symmetry 
breaking this interaction term will lead to mixing between $D_{1}$ and S. Note that $D_{1}$ and $\mathrm{S}$ carries the same charge and color assignments which is necessary so that they could mix after EWSB. The above higgs-LQ-DQ interaction term violates both baryon number and lepton number by one unit $(\delta B=-\delta L=1)$. Here we are implicitly assuming that the breaking of baryon number symmetry is first communicated to the scalar sector of the high energy theory which in turn communicates it to other sectors e.g the Yukawa sector. Recall that in the SM baryon number conservation is realized as an accidental global symmetry [4]. By that we mean that given the particle content and the gauge group $S U(3)_{c} \times S U(2)_{l} \times U(1)_{y}$ of the $\mathrm{SM}$ it is not possible to write a renormalizable and gauge invariant interaction term that violates baryon number. No adhoc global symmetry is required to explain the near abscence of proton decay. However as we have seen above that if we keep the gauge group the same but allow additional particles like color triplet scalars then it is possible to write down a renormalizable and gauge invariant interaction term that violates baryon number.

We shall now show that the mixing between $D_{1}$ and S induced by $\phi$ can lead to proton decay in the " $\nu+$ any channel" where any refers to a positively charged non-strange meson. Consider the full scalar potential involving D and S

$$
\begin{aligned}
V(D, S) & =\mu_{1}^{2} D^{+} D+\mu_{2}^{2} S^{+} S+\lambda_{1}\left(D^{+} D\right)^{2}+\lambda_{2}\left(S^{+} S\right)^{2} \\
& +\lambda_{1}^{\prime}\left(D^{+} D\right)\left(\phi^{+} \phi\right)+\lambda_{2}^{\prime}\left(S^{+} S\right)\left(\phi^{+} \phi\right)+\left(k_{1} D^{+} \phi_{c} S+\text { h.c. }\right)
\end{aligned}
$$

The quartic scalar interactions are not relevant to our present work. $\mu_{1}^{2}$ and $\mu_{2}^{2}$ are the mass parameters associated with the gauge eigenstates D and S. After EWSB the trilinear interaction term between $\phi, \mathrm{D}$ and $\mathrm{S}$ induces a mixing between $D_{1}$ and $\mathrm{S}$. It can be shown that the eigenvalues of the mass squared matrix $M_{D_{1} S}^{2}$ are given by

$$
\lambda_{+}=M_{D_{1}}^{2}=\frac{1}{2}\left(\mu_{1}^{2}+\mu_{2}^{2}\right)+\frac{1}{2} \sqrt{\epsilon^{2}+2 k_{1}^{2} v^{2}} .
$$

and

$$
\lambda_{-}=M_{S}^{2}=\frac{1}{2}\left(\mu_{1}^{2}+\mu_{2}^{2}\right)-\frac{1}{2} \sqrt{\epsilon^{2}+2 k_{1}^{2} v^{2}}
$$


where $\epsilon=\mu_{1}^{2}-\mu_{2}^{2}$. The physical states correponding to $\lambda_{ \pm}$are given by $D_{1}^{\prime}=D_{1} \cos \theta-$ $S \sin \theta$ and $S^{\prime}=D_{1} \sin \theta+S \cos \theta$. Here $\cos \theta=\frac{\sqrt{2} k_{1} v}{\left[2 k_{1}^{2} v^{2}+\left(\sqrt{\epsilon^{2}+2 k_{1}^{2} v^{2}}-\epsilon\right)^{2}\right]^{\frac{1}{2}}}$.

The Yukawa couplings of the LQ and the DQ written in terms of mass eigenstates are given by

$$
\begin{aligned}
L_{y} & =g_{R}\left[\bar{\nu}_{L}\left(D_{1}^{\prime} \cos \theta+S^{\prime} \sin \theta+\bar{e}_{L} D_{2}\right] d_{R}\right. \\
& +\epsilon_{i j k} g_{R}^{\prime} \bar{d}_{R i} u_{R j}^{c}\left[S_{k}^{\prime} \cos \theta-D_{1 k}^{\prime} \sin \theta+\text { h.c. }\right]
\end{aligned}
$$

- The above Yukawa Lagrangian does lead to proton decay in the " $\nu+$ any" channel via the exchange of D and S particles. The effective four fermion Lagrangian for this decay is given by

$$
L_{e f f}=g_{R} g_{R}^{\prime} \sin \theta \cos \theta \epsilon_{i j k}\left(\bar{\nu}_{L} d_{R k}\right)\left(\bar{u}_{R j}^{c} d_{R i}\right)\left(\frac{1}{M_{S}^{2}}-\frac{1}{M_{D}^{2}}\right)+h . c .
$$

This is the effective Lagrangian at a scale $M^{2}=M_{D}^{2} \approx M_{S}^{2} \approx(250 G e v)^{2}$. In order to use it for proton decay it has to be renormalized down to a scale $\mu^{2}=(1 G e v)^{2}$ under the unbroken QCD and EM interactions. The EM corrections are small because the coupling itself is small. The QCD corrections are not that large either because $\ln \frac{M^{2}}{\mu^{2}}$ is not large in our case. It can be shown that the proton decay rate arising from the above effective Lagrangian is given by

$$
\Gamma\left(p \rightarrow \nu_{e}+\text { any }\right) \approx \frac{1}{17 \pi^{2} R^{3}} G_{e f f}^{2} m_{q}^{2} A^{2}\left(\frac{M}{\mu}\right)
$$

Here $A\left(\frac{M}{\mu}\right)$ includes the effects of renormalization group evolution from $\mathrm{M}$ to $\mu$. We shall assume it to be of order one. $R=\frac{3}{4} \mathrm{fm}, m_{q} \approx \frac{1}{3}$ Gev and $G_{e f f}=\frac{1}{2 \sqrt{2}} g_{R} g_{R}^{\prime} \sin \theta \cos \theta\left(\frac{1}{M_{S}^{2}}-\right.$ $\left.\frac{1}{M_{D}^{2}}\right)$. The present lower bound on $\tau(p \rightarrow \nu+$ any $)$ is $25 \times 10^{30} \mathrm{yr}$ [5]. It is clear from the expression of $G_{\text {eff }}$ that the tight constraint of proton lifetime can be satisfied either with $\sin \theta \ll 1$ or $\cos \theta \ll 1$. It can be shown that for $\sin \theta \ll 1$ we require $k_{1} v \ll \sqrt{2} \epsilon$. On the other hand for $\cos \theta \ll 1$ we need $\sqrt{2} k_{1} v \ll\left(\sqrt{\epsilon^{2}+2 k_{1}^{2} v^{2}}-\epsilon\right)$. For $M_{S}=200 \mathrm{Gev}$ and $M_{D}=300 \mathrm{Gev}$ [6] the product combination $g_{R} g_{R}^{\prime} \sin \theta \cos \theta$ 
must be less than $3 \times 10^{-26}$ in order that the decay rate in eqn(7) is consistent with the proton lifetime. If we assume that the Yukawa couplings $g_{R}$ and $g_{R}^{\prime}$ are of the order of .1 , then for $\sin \theta \ll 1$ we must adjust the parameters of the Lagrangian so as to satisfy $k_{1} v<4 \times 10^{-24} \epsilon$. On the other hand for $\cos \theta \ll 1$ the parameters must be adjusted so as to satisfy $\sqrt{2} k_{1} v<3 \times 10^{-24}\left(\sqrt{\epsilon^{2}+2 k_{1}^{2} v^{2}}-\epsilon\right)$. These constraints may not be stable against radiative corrections requiring extreme fine tuning to each order in loop expansion. We would like to reiterate that there can be several different species of LQ's as well as DQ's. However the mixing between $D_{1}$ and $\mathrm{S}$ and hence proton decay occurs only for specific $S U(3)_{c} \times S U(2)_{l} \times U(1)_{y}$ assignments of the LQ and the DQ. Unless these assignments are achieved there is no mixing between the LQ and DQ and hence no contribution to proton decay.

Having shown that higgs induced mixing between the doublet leptoquark D and the singlet diquark S does lead to proton decay we shall now present another concrete example where the same phenomenon also takes place. An EW triplet diquark $T_{a k}$ (a is the $S U(2)_{l}$ index and $\mathrm{k}$ the color index) can also mix with the doublet leptoquark $\mathrm{D}$ and contribute to both proton decay and neutron decay. Let the $S U(3)_{c} \times S U(2)_{l} \times U(1)_{y}$ assignments of $T_{a k}$ be given by $\left(3,3,-\frac{1}{3}\right)$. The triplet diquark $T_{a k}$ can couple to LH quark pair according to the following Lagrangian:

$$
\begin{aligned}
L_{y}^{\prime} & =g_{R}^{\prime \prime} \epsilon_{i j k} \bar{q}_{L i}^{c} \tau_{a} i \tau_{2} T_{a k}+\text { h.c. } \\
& =g_{R}^{\prime \prime} \epsilon_{i j k}\left[-\sqrt{2} \bar{u}_{L i}^{c} u_{L j} T_{-k}+\sqrt{2} \bar{d}_{L i}^{c} d_{L j} T_{+k}+\left(\bar{u}_{L i}^{c} d_{L j}+\bar{d}_{L i}^{c} u_{L j}\right) T_{0 k}\right]
\end{aligned}
$$

where $T_{+k}=\frac{T_{1 k}+i T_{2 k}}{\sqrt{2}}, T_{-k}=\frac{T_{1 k}-i T_{2 k}}{\sqrt{2}}$ and $T_{0 k}=T_{3 k} . T_{+k}$ and $T_{-k}$ carry electromagnetic charges of $\frac{2}{3}$ and $-\frac{4}{3}$ units respectively. Note that $T_{-k}$ is not the antiparticle of $T_{+k}$ since they carry different electromagnetic charges. The gauge invariant Higgs-LQ-DQ interaction term in this case will be given by

$$
\begin{aligned}
L_{s}^{\prime} & =k_{2}\left(D^{+} \tau_{a} \phi_{c}\right) T_{a}^{*}+h . c . \\
& =k_{2} \frac{v+h}{\sqrt{2}}\left[D_{2}^{+} T_{+}^{*}+D_{1}^{+} T_{0}^{*}+\text { h.c. }\right]
\end{aligned}
$$


The mixing between $D_{1}$ and $T_{0}^{*}$ leads to $p \rightarrow \pi^{+} \nu$ and the mixing between $D_{2}$ and $T_{+}^{*}$ leads to $n \rightarrow \pi^{+} e^{-}$both of which violate baryon number.

The low energy effective Lagrangian will also contain trilinear Higgs-LQ-LQ and HiggsDQ-DQ interaction terms. Afetr EWSB these terms give rise to mixing between different multiplets of LQ and DQ. Such interaction terms do not violate baryon number but they could violate lepton number. Of particular importance is the Higgs-LQ-LQ ineraction for a pair of chiral leptoquarks belonging to different weak $\mathrm{SU}(2)$ multiplets. The mixing between two different LQ multiplets will give rise to helicity unsuppressed contribution to $\pi^{-} \rightarrow e^{-} \bar{\nu}_{e}$ The helicity suppressed SM contribution to $\pi^{-} \rightarrow e^{-} \bar{\nu}_{e}$ is in excellent agreement with the experimental data. Therefore any helicity unsuppressed contribution arising from new physics must be strongly constrained. This leads to stringent bounds on the mixing parameter between the two LQ multiplets. The mixing between different LQ multiplets can also lead to majorana mass matrix for neutrinos. A complete discussion of these topics can be found in Ref. [7] and will not be taken up here.

To conclude in this report we have shown that the usual assumption of low energy phenomenology that LQ's couple only to q-l pairs and DQ's couple only to quark pairs is not sufficient to stabilize the proton. We have given two specific examples where the Higgs-LQDQ interaction term induces a mixing between the LQ and DQ after EWSB. This mixing reintroduces the troublesome couplings for the LQ and the DQ and leads to proton decay. We find that in order to be consistent with the bound on proton lifetime the parameters of the Lagrangian must either satisfy $k_{1} v<4 \times 10^{-24} \epsilon$ or $\sqrt{2} k_{1} v<3 \times 10^{-24}\left(\sqrt{\epsilon^{2}+2 k_{1}^{2} v^{2}}-\epsilon\right)$. In deriving these constraints we have assumed that $g_{R} \approx g_{R}^{\prime} \approx .1, M_{D_{1}}=300 \mathrm{Gev}$ and $M_{S}=200 \mathrm{Gev}$. These constraints may not be stable against radiative corrections requiring extreme fine tuning of the parameters to each order in loop expansion. This naturalness problem can perhaps be resloved by means of some new symmetry that remains unbroken in the low energy theory and prevents the Higgs-LQ-DQ interaction term from occuring in the effective Lagrangian. 


\section{References}

1. W. Buchmuller Acta Phys. Austriaca, Suppl 27, 517 (1985); W. Buchmuller and D. Wyler, Phys. Lett. B 177, 377 (1986).

2. E. Farhi and L. Susskind, Phys. Rep. 74, 277 (1981); K. Lane and M. V. Ramanna, Phys. Rev. D 44, 2678 (1991).

3. J. Hewett and T. Rizzo, Phys. Rep. 183, 193 (1989).

4. A. Cohen, A. Nelson and D. Kaplan, Phys. Lett. B 388, 588 (1996).

5. Review of Particle Properties Euro. Phy. Jour. C3, 50 (1998).

6. The values of $M_{S}$ and $M_{D}$ assumed by us are consistent with the latest bounds on LQ and DQ masses: Euro. Phys. Jour. C3, 260 (1998); B. Abbot et al. (DO Collaboration), Phys. Rev. Lett. 80, 2051 (1998); F. Abe et al. (CDF Collaboration), Phys. Rev. D 55, R5263 (1997).

7. M. Hirsch et al., Phys. Rev. D 54, 4207(1996); Phys. Lett. B 378, 17 (1996); U. Mahanta, MRI-PHY/990930, hep-ph/9909518. 